CRAL-2006. Chemodynamics: From First Stars to Local Galaxies

E. Emsellem, H. Wozniak, G. Massacrier, J.-F. Gonzalez, J. Devriendt and N. Champavert (eds)

EAS Publications Series, Vol. 24 (2007)

\title{
MASSIVE STARS: THEIR CONTRIBUTION TO ENERGY AND ELEMENT BUDGET IN CHEMO-DYNAMICAL GALAXY EVOLUTION
}

\author{
Gerhard Hensler ${ }^{1}$
}

\begin{abstract}
Here results of numerical radiation hydrodynamical simulations are presented which explore the energetic impact of massive stars on the interstellar medium. We study the evolution of the ambient gas around isolated massive stars in the mass range between 15 and $85 \mathrm{M}_{\odot}$ in order to analyze the formation of structures and further the transfer and deposit of the stellar wind and radiation energy into the circumstellar medium until the stars explode as a supernovae. The derived energy transfer efficiencies are much smaller than analytically estimated and should be inserted into chemo-dynamical evolutionary models of galaxies as appropriate parameter values. As an additional issue the element release in the Wolf-Rayet phases and its detectability have been investigated for comparison with observations.
\end{abstract}

\section{Introduction}

Besides external effects structure and evolution of galaxies is primarily determined by the energy budget of its interstellar medium (ISM). Reasonably, also the ISM structure and both its radiative and kinetic energy contents are determined by the energy deposit of different sources, on the one hand, and by the energy loss by means of radiative cooling on the other. Mainly massive stars contribute significantly to this structure formation like e.g. cavities, holes, and chimneys in the Hi gas and superbubbles of hot gas (Recchi \& Hensler 2006). On large scales the energy release by massive stars triggers the matter circulations via galactic outflows from a gaseous disk and galactic winds (Recchi \& Hensler, this volume). By this, also the chemical evolution is affected thru the loss of metal-enriched gas from a galaxy (see e.g. Recchi et al. 2006).

Supernova (SN) explosions as an immediate consequence of star formation (SF) stir up the ISM by the expansion of hot bubbles, deposit turbulent energy into

\footnotetext{
${ }^{1}$ Institute of Astronomy, University of Vienna, Türkenschanzstr. 17, A-1180 Vienna, Austria, e-mail: hensler@astro.univie.ac.at
} 
the ISM and, thereby, regulate the SF again (Hensler \& Rieschick 2002). This negative energy feedback is enhanced at low gravitation because the SN energy exceeds easily the galactic binding energy and drives a galactic wind. Vice versa, SN and stellar wind-driven bubbles sweep up surrounding gas and can, by this, excite SF self-propagation as a positive feedback mechanism (e.g. Ehlerova et al. 1997, Fukuda \& Hanawa 2000).

Since the numerical treatment of the galactic chemo-dynamical evolution, although when it deals with two gas phases, cannot resolve the ISM spatially sufficiently, for a detailed consideration of small-scale processes the chemo-dynamical modeling has to apply parametrizations of plasmaphysical processes. It should, however, be repeatedly emphasized that an appropriate chemo-dynamical description cannot vary those parameters arbitrarily, because they rely on results from theoretical, numerical, and/or empirical studies and are by this strongly constrained. This has been described and applied to different levels of chemodynamical models (for an overview see Hensler, 2003 and references therein, and more recently Hensler et al., 2004, Harfst et al., 2006).

As an example, analytical consideration of heating and cooling balance by Köppen et al. (1995) making use of the cooling by collisionally excited radiation (Böhringer \& Hensler 1989) yields that the SF rate under reasonable ISM states is dependent on the square of the gas density with a slight influence of the heating efficiency. This fact is surprising: although the ionizing radiative fluxes of massive stars are pretty well known from static stellar atmosphere models (see e.g. Thompson 1984) or more sophisticated wind atmospheres (Kudritzki \& Puls 2000), the fraction of the radiative energy that is thermalizing the gas is uncertain.

In addition, massive stars act also dynamically on their surrounding ISM by a strong stellar wind (Kudritzki \& Puls 2000). Although the stellar wind power $L_{w}=\frac{1}{2} \dot{M}_{w} v_{w}^{2}$ with the mass-loss rate $\left(\dot{M}_{w}\right)$ and the terminal wind velocity $\left(v_{w}\right)$ can be easily evaluated from model and observational values, the fraction of the wind luminosity which is transferred e.g. into thermal energy, the so-called thermal energy transfer efficiency, or into turbulent energy is not obvious from principles.

\section{Theory of radiative and stellar wind bubbles}

Within the framework of the simplest theoretical approach an $\mathrm{O}$ star suddenly "turns on" in a constant density medium at rest and begins to ionize its surroundings. The reaction of the medium to the stellar photons is well-known and has been described in detail in standard textbooks. With the particular knowledge of the time evolution of the ionization front at distance from a star of age $\tau$ and with respect to the initial Strömgren radius $R_{s}$ which depends on the Lyman continuum photon luminosity, the Hi number density of the ambient medium, and the isothermal sound speed of the ionized gas Lasker (1967) derived the kinetic energy of the expanding swept-up shell $E_{\mathrm{k}}$, the ionization energy stored in the HII region $E_{\mathrm{i}}$, and the thermal energy of warm gas in the Hil region $E_{\mathrm{t}}$ (see also Freyer et al. 2003, hereafter: Paper I). As an appropriate approximation the temperature in the HII region needed in the set of formulae can be set to $8000 \mathrm{~K}$. 
Also for stellar wind bubbles (SWBs) the radial structure can be analytically derived (see Weaver et al. 1977 and Paper I) and, furthermore, also the kinetic energy as $E_{\mathrm{k}}=\frac{3}{11} L_{\mathrm{w}} \tau$ and the thermal energy of the hot gas as $E_{\mathrm{t}}=\frac{5}{11} L_{\mathrm{w}} \tau$.

Besides heat conduction and cooling in the hot bubble here also neglected is the fact that part of the thermal energy might be used for collisional ionization of gas. Thus, the results for $E_{\mathrm{k}}$ and $E_{\mathrm{t}}$ are upper limits.

Because these analytical derivations cannot account for aspherical structure formation and turbulence within the SWB which both consume stellar energy and alter the energy fractions numerical models are needed to investigate the massive stellar energy deposit into the ISM. For a comprehensive tabulation and comparison of former models the interested reader is referred to Paper I.

\section{The model}

The numerical code applied for this purpose and used to obtain the results presented here is described in Paper I. The hydrodynamical equations are solved together with the transfer of H-ionizing photons on a $2 \mathrm{~d}$ cylindrical grid. The timedependent ionization and recombination of hydrogen is calculated each timestep, and we carefully take stock of all the important energy exchange processes in the system. A comprehensive description of the algorithm is given in Yorke \& Kaisig (1995), Yorke \& Welz (1996), and in Paper I.

This code is applied to the evolution of massive stars with 4 different masses: $15 \mathrm{M}_{\odot}$ (Kroeger, Freyer, Hensler \& Yorke, in prep. as Paper IV of this series), $35 \mathrm{M}_{\odot}$ (Freyer et al. 2006 Paper II of this series), $60 \mathrm{M}_{\odot}$ (Paper I), and $85 \mathrm{M}_{\odot}$ (Kroeger et al. 2006b: Paper III of this series). Because we started the project also at first for comparison with the $60 \mathrm{M}_{\odot}$ model published by Garcia-Segura et al. (1996a) we adopted basically the same stellar parameters applied by them from stellar evolutionary models and from observations. As initial conditions of the ambient medium we also use the same undisturbed background gas layer as Garcia-Segura et al. with HI number density $n_{0}=20 \mathrm{~cm}^{-3}$ and temperature $T_{0}=$ $200 \mathrm{~K}$. This yields a thermal pressure "typical" for the ISM at the solar galactocentric distance. Also the $35 \mathrm{M}_{\odot}$ model was aimed for comparison with GarciaSegura et al. (1996b) and used their stellar parameters along its life. Because from this group no evolutionary tracks and stellar parameters of other stellar masses became available yet, however, for the $15 \mathrm{M}_{\odot}$ and the $85 \mathrm{M}_{\odot}$ models we were compelled to apply stellar evolutionary models from Schaller et al. (1992). Although this makes the sample inhomogeneous, the extensive computational time necessary for the models only allowed until now comparison of the two $60 \mathrm{M}_{\odot}$ star model sources. As a first result, we obtained for the Schaller et al. model a by one order of magnitude weaker wind luminosity (Hensler, Kroeger, \& Freyer, in prep.). Furthermore, the evolutionary sequence of Wolf-Rayet (WR) and Luminous Blue Variable (LBV) phases are reverse from one group to the other in the sense that the WR phase precedes the LBV phase in the Langer models.

In our models the stellar parameters $\dot{M}_{w}, v_{w}$, effective temperature $\left(T_{\text {eff }}\right)$, and photon luminosity in the Lyman continuum $\left(L_{\mathrm{LyC}}\right)$ are time-dependent boundary 

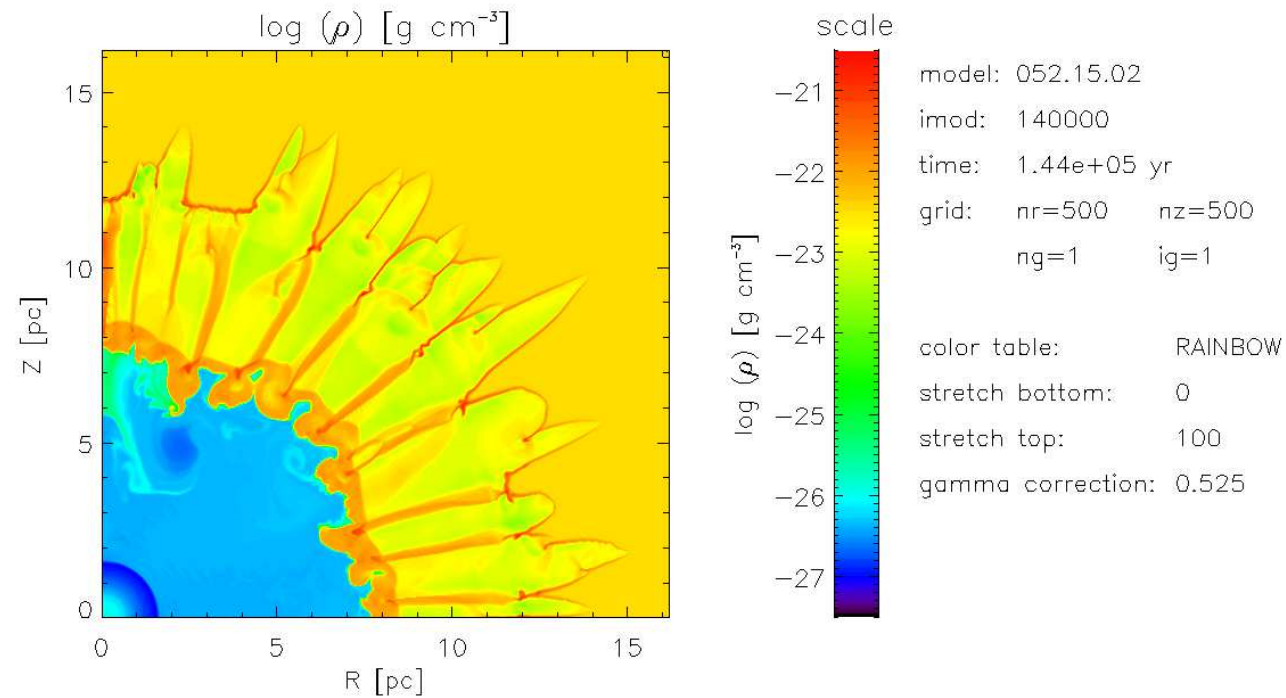

Fig. 1. Density plot of the wind-blow and radiation-driven bubble around a $60 \mathrm{M}_{\odot}$ main-sequence star at an age of 140000 years.

conditions which drive and govern the evolution of the circumstellar gas.

We start our calculations with the sudden turn-on of the zero-age main-sequence stellar radiation field and stellar wind. For the surrounding gas we neglect any photo-dissociation of molecular content here which would in reality precede the photo-ionization stage (e.g. Kroeger, Hensler, \& Freyer, in prep. as Paper V of this series).

\section{Results}

As a first result, the winds structure the surrounding gas by dynamical shock front instabilities during its vehement expansion. These lead to the formation of condensations within the wind shell which, on the other hand, have higher optical depth for the ionizing photons. Finger-like extentions of the HiI region surrounding the SWB are most characteristicly formed for the $60 \mathrm{M}_{\odot}$ model (see Fig.1) from photons penetrating deeper into the ambient ISM. In addition, photoevaporation peels off the inner boundary of the wind shell and drives mass loading and turbulence.

Another relevant question addressedto these models is their energy deposit into the ISM. Here we can only briefly mention a few major issues. Over the whole evolution one can trace the energy released in different forms as kinetic $E_{\mathrm{k}}$, photo-ionizing $E_{\mathrm{i}}$, and thermal $E_{\mathrm{t}}$ energies, respectively, by book keeping so that at every stellar age $\tau$ each integrated energy form released can be compared with that inherently available in the computational domain. The ratio of deposited stellar energy to the increase in one form compared to the initial state can be 


\section{energy transfer efficiency $\varepsilon$}

\section{$\varepsilon \cdot 10^{4}$}

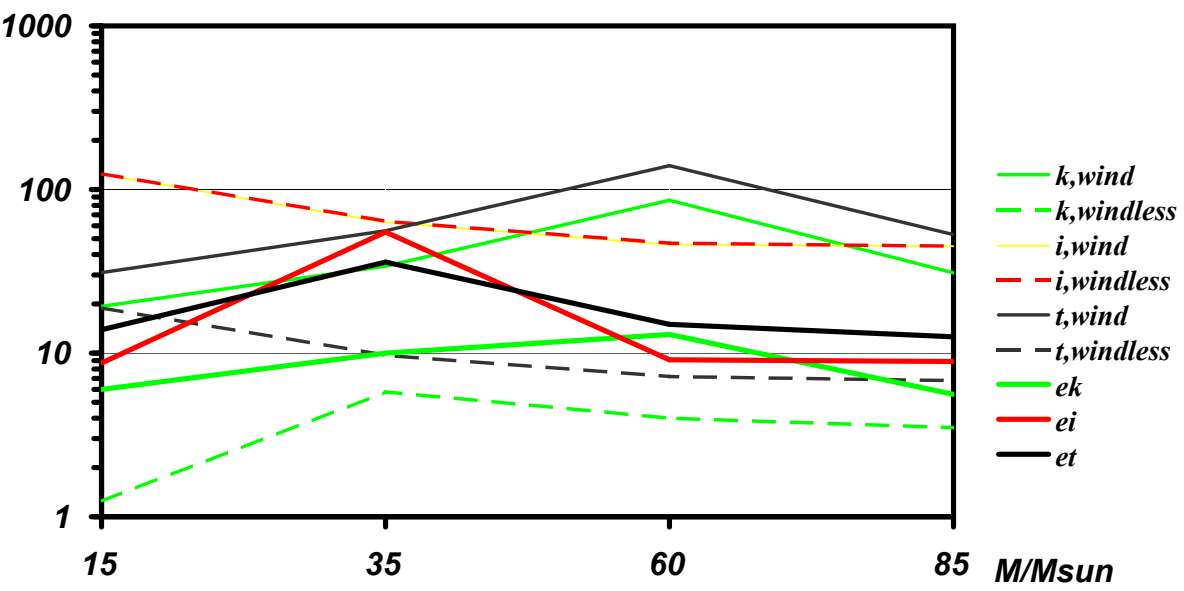

Fig. 2. Energy transfer efficiencies $\varepsilon_{\mathrm{k}}, \varepsilon_{\mathrm{i}}$, and $\varepsilon_{\mathrm{t}}$ for kinetic, ionizing photon, and thermal energies for the $15,35,60$, and $85 \mathrm{M}_{\odot}$ models. $\mathrm{k}$, windless and $\mathrm{k}$, wind as well as for $\mathrm{i}$ and $\mathrm{t}$, respectively, represent the analytical values according to equ 4.1 and its extention by $L_{\mathrm{w}}$, while $e k, e i$, et give the efficiency values at the end of the stellar lifetimes.

defined as an energy transfer efficiency $\varepsilon$. As the simplest approach, $\varepsilon$ for pure Lyman continuum radiation into the other energy forms is then descibed by the analytical approach of

$$
\varepsilon=\frac{E}{\tau\left\langle L_{\mathrm{LyC}}\right\rangle},
$$

where $E$ can be any of $E_{\mathrm{k}}, E_{\mathrm{i}}$, and $E_{\mathrm{t}}$, respectively. For radiation plus wind energy this nominator has to be extended accordingly to $\left(\left\langle L_{\mathrm{LyC}}\right\rangle+\left\langle L_{\mathrm{w}}\right\rangle\right)$.

These $\varepsilon$ values have been derived for all four massive star models and their energy forms (Hensler, Freyer, \& Kroeger, 2007, in prep. as Paper VI of this series) and are exhibited in Fig.2. Not surprisingly, the windless values are below the wind models except the ionization efficiency $\varepsilon_{\mathrm{i}}$. Nevertheless, the average $\varepsilon$ only exceed one percent for the thermal efficiency of the $60 \mathrm{M}_{\odot}$ wind model. As already mentioned, there the structure formation is strongest and might be reduced applying the Schaller et al. (1992) model.

The unexpected decrease of $\varepsilon_{\mathrm{i}}$ (the upper dashed curve in Fig.2) with increasing stellar mass is caused by the growing strength of stellar winds with mass that have the dynamical effect to compress the ambient shells much stronger so that the recombination is enhanced. This means, ionization energy is more efficiently lost for larger stellar masses.

Comparing the windless i.e. pure photo-ionizing models with analytical results e.g. by Lasker (1967) of about one percent, our efficiencies fall short by more than one order of magnitude even to below 0.1 percent (Fig.2). $\varepsilon_{\mathrm{t}}$ reaches more than 
one percent only for $60 \mathrm{M}_{\odot}$. As another important issue, it should be mentioned that the final efficiencies (ek,ei, et in Fig.2) are located significantly below the values integrated over the stellar life because of the waeker wind at the last stages.

The efficiencies derived from our models are much less than expected and of fundamental importance for the energy budget of galaxies during their evolution. They should be easily inserted into chemo-dynamical evolutionary codes because integrating the stellar energy release by massive stars over their concedingly short lifetimes without taking detailed heating vs. cooling processes into account would falsify the treatment of the ISM significantly. A comprehensive discussion is in preparation as Paper VI.

Finally, a byproduct of these studies should be mentioned, namely, that the elements release in the WR phase of most massive stars, in particular of carbon, although at first incorporated into the hot SWB gas are enabled to cool by mixing effects and become observable as warm ionized His gas (Kroeger et al. 2006a).

The author thanks Danica Kroeger and Tim Freyer for their strong engagement to this project and the organizers for this gorgeous and most exciting conference.

\section{References}

Böhringer, H., \& Hensler, G. 1989, A\&A, 215, 147

Ehlerova, S., Palous, J., Theis, C., \& Hensler, G. 1997, A\&A, 328, 111

Freyer, T., Hensler, G., \& Yorke, H.W., 2003, ApJ, 594, 888 (Paper II)

Freyer, T., Hensler, G., \& Yorke, H.W., 2006, ApJ, 638, 262 (Paper I)

Fukuda, N. \& Hanawa, T. 2000, ApJ, 533, 911

García-Segura, G., Mac Low, M.-M., \& Langer, N. 1996a, A\&A, 305, 229

García-Segura, G., Langer, N., \& Mac Low, M.-M. 1996b, A\&A, 316, 133

Harfst, S., Theis, C., \& Hensler, G. 2006, A\&A, 499, 509

Hensler, G. 2003, in CNO in the Universe, eds. C. Charbonnel et al., ASP Conf. Ser. Vol. 304, 371

Hensler, G. \& Rieschick, A. 2002, in Modes of Star Formation, eds. E.K. Grebel \& W. Brandner, ASP Conf. Ser. Vol. 285, p. 341

Hensler, G., Theis, Ch., \& Gallagher, J.S. 2004, A\&A, 426, 25

Köppen, J., Theis, C., \& Hensler, G. 1995, A\&A, 296, 99

Kroeger D., Hensler, G., \& Freyer T., 2006a, A\&A, 450, L5

Kroeger D., Freyer T., Hensler, G., \& Yorke, H.W. 2006b, A\&A, submitted (Paper III)

Kudritzk, R.P. \& Puls, J. 2000, ARA\&A, 38, 613

Lasker, B.M. 1967, ApJ, 149, 23

Recchi, S., \& Hensler, G. 2006, A\&A, 445, L39

Recchi, S., Hensler, G., Angeretti, L., \& Matteucci, F. 2006, A\&A, 445, 875

Schaller, G., Schaerer, D., Maeder, A., \& Meynet, G. 1992, A\&AS, 96, 269

Thompson, R.I. 1984, ApJ, 283, 165

Weaver, R., McCray, R., Castor, J., et al. 1977, ApJ, 218, 377

Yorke, H. W., \& Kaisig, M. 1995, Comput. Phys. Commun., 89, 29

Yorke, H. W., \& Welz, A. 1996, A\&A, 315, 555 